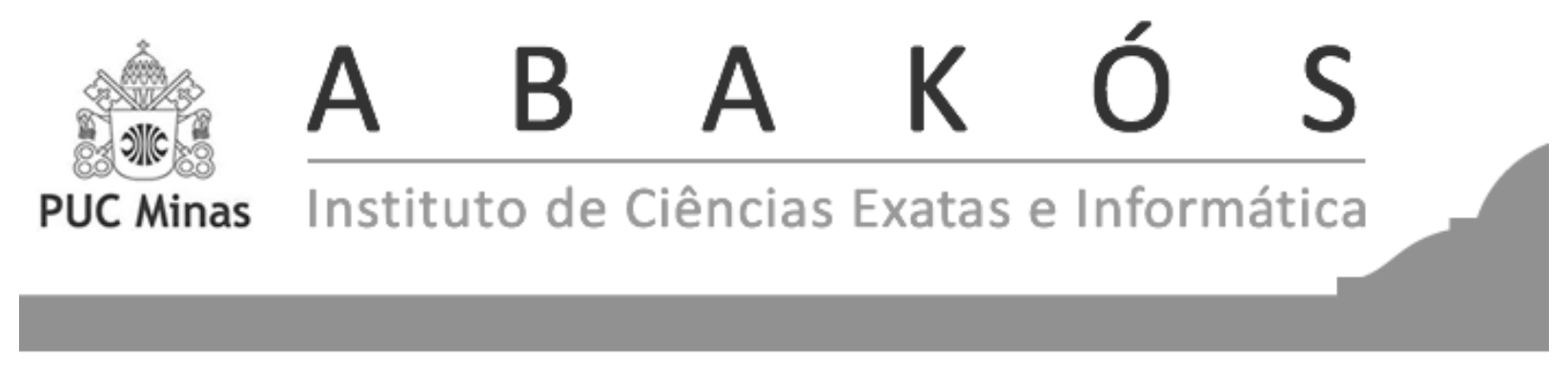

cc) (1)

\title{
Caracterizações do Pensamento Algébrico na Escola Básica*
}

Characterizing Algebraic Thinking in Elementary School

\author{
Marcela da Silva Caetano Rodrigues ${ }^{1}$ \\ Márcia Maria Fusaro Pinto ${ }^{2}$
}

\begin{abstract}
Resumo
Este artigo é resultado de um estudo sobre caracterizações do que vem sendo entendido como Pensamento Algébrico na escola básica. Apresentamos autores que são referências constantes em pesquisas sobre o tema, e discutimos os elementos teóricos elaborados por eles, com resultados e exemplos da utilização de tais noções em investigações empíricas. Concluímos apresentando nosso próprio entendimento sobre Pensamento Algébrico na escola básica, com reflexões sobre quando, como e por que elaborar atividades em sala de aula da escola básica que tenham por objetivo o seu desenvolvimento, e que promovam habilidades que serão posteriormente requeridas em conteúdos mais avançados da Matemática.
\end{abstract}

Palavras-chave: Pensamento Algébrico. Ensino de Matemática. Matemática. Álgebra na Escola Básica.

\footnotetext{
* Submetido em 21/01/2019 - Aceito em 11/02/2020

${ }^{1}$ Programa de Pós-graduação em Ensino e História da Matemática e da Física (PEMAT-UFRJ), Secretária Municipal de Educação (SME-Mangaratiba/RJ e SME-Barra do Piraí/RJ), Brasil - marcelasc.marcelasc@ gmail.com ${ }^{2}$ Programa de Pós-Graduação em Ensino e História da Matemática e da Física (PEMAT-UFRJ), Brasil marciafusaro@gmail.com
} 


\begin{abstract}
This paper is the result of a study on characterizations of what has been understood as Algebraic Thinking in elementary and secondary schools. We present authors who are constant references in research on the theme, discussing the theoretical elements elaborated by them, bringing results and examples of the use of such notions in empirical investigations. We conclude by presenting our own understanding of Algebraic Thinking in elementary school, reflecting on when, how and why to develop classroom activities that aim its development by promoting skills that will later be required in more advanced mathematics topics.
\end{abstract}

Keywords: Algebraic Thinking. Mathematics Education. Algebra in elementary school. 


\section{INTRODUÇÃO}

Caracterizações do Pensamento Algébrico na escola básica têm sido tema de debates e de pesquisas no campo da Educação Matemática, no âmbito nacional e internacional. Elaboramos este artigo retomando alguns entendimentos sobre o Pensamento Algébrico na escola básica compartilhados na literatura desse campo do saber. Priorizamos autores que, com frequência, têm sido referências em pesquisas empíricas sobre o tema no Brasil. Concluímos, voltando nosso olhar para os resultados dessas investigações, refletindo sobre como o Pensamento Algébrico pode ser desenvolvido, bem como quando é adequado trabalhá-lo na escola básica. Os documentos aprovados, em 1998, pelo Ministério da Educação (MEC) como diretrizes para a educação na escola básica no país, os Parâmetros Curriculares Nacionais (PCN), são retomados ao destacarmos as diretrizes que se referem ao desenvolvimento do Pensamento Algébrico na escola básica, relacionando-as à literatura de pesquisa estudada.

Antecedendo tal apresentação, esclarecemos nosso entendimento sobre as expressões "linguagem simbólica", "formalização" e "ensino mecânico", utilizadas ao embasarmos nossa crítica a um ensino de Álgebra na escola básica que consideramos mecanizado.

Ao utilizarmos a expressão "linguagem simbólica", estamos nos referindo não somente ao uso de incógnitas e variáveis, mas também à utilização de simbologias, signos e sinais, quaisquer que sejam eles, para expressão do pensamento, comunicação e interação entre pessoas. No dicionário Houaiss e Villar (2001), o termo simbólico define o que é próprio do símbolo, que não é uma reprodução da realidade; mas sim, aquilo que, por um princípio de analogia formal ou de outra natureza, substitui ou sugere algo (HOUAISS; VILLAR, 2001). Na linguagem simbólica, os signos ou símbolos aludem de forma abstrata e convencional às realidades a que se referem. Por exemplo, no caso das luzes dos semáforos, o verde corresponde a "prossiga", e o vermelho a "pare". Desse modo, se considerarmos, por exemplo, os três modos de representação do conhecimento propostos em Bruner (1996) - de domínio da ação, icônico e simbólico, nosso entendimento de "linguagem simbólica" irá incluir todos esses três. Para Bruner, o pensamento simbólico, representado pelo terceiro modo de representação, não substitui os representados pelos outros modos. Corrobora esse entendimento uma noção de "linguagem simbólica" que amplia seu escopo para contemplar formas de comunicação por meio de ações (gestos, por exemplo), ícones (diagramas, por exemplo) e símbolos, que incluem os símbolos matemáticos algébricos, como estamos propondo.

Com o termo "formalização", referimo-nos ao processo pelo qual o terceiro modo de representação proposto em Bruner (1996) é gradativamente incorporado à escrita e à comunicação utilizadas em Matemática na escola básica. Reflete-se no uso crescente de incógnitas, variáveis e símbolos operacionais.

Ao falarmos sobre "ensino mecânico" da Matemática, estaremos nos referindo ao "[... ] ensino da Matemática de forma tradicional, como uma simples transmissão de conhecimento através de técnicas desprovidas de significado e a repetição do a lgoritmo s em s entido algum [...]" (OLIVEIRA; LAUDARES, 2015, p.2). Isso não significa desconsiderar a importância da 
"familiaridade com símbolos individuais do sistema e com as convenções sintáticas das configurações simbólicas aceitas", nem das "regras ou procedimentos para resolver problemas matemáticos" (HIEBERT, 1986, p.7) ou seja, o ensino de algoritmos, definições, provas e demonstrações tem importância sim, e é relevante para a aprendizagem dos alunos. Significa destacar a importância também para este tipo de conhecimento de que "todos os tipos de informação estejam conectados a alguma rede" de conceitos (HIEBERT, 1986, p.4), pois, caso isso não aconteça, "os alunos podem produzir respostas, mas não entendem o que eles estão fazendo" (HIEBERT, 1986, p.9). Quando apresentado da forma que denominamos "mecânica", o ensino de Álgebra:

[...] não possibilita ao aluno fazer conexões e pensar de forma autônoma e nem facilita a compreensão dos conceitos e procedimentos estabelecidos pela Álgebra. [...] Se o aluno não é capaz de apropriar-se dos conceitos algébricos ele não desperta o prazer de aprendê-los. (OLIVEIRA; LAUDARES, 2015, p. 3).

Entendemos que desenvolver o Pensamento Algébrico é possibilitar, de modo significativo para o aluno, o estabelecimento de conexões entre os diversos conceitos e procedimentos da Álgebra que estão sendo estudados. Nossa intenção, neste estudo, é discutir tal desenvolvimento, refletindo s obre o e nsino d a Á lgebra n a e scola b á sica. N o que s e s e gue, buscamos referências para elaborar nossa própria caracterização de Pensamento Algébrico, a partir de elementos comuns que diversos autores que tratam do tema utilizam para elaborar sua noção de Pensamento Algébrico na escola básica.

\section{CARACTERIZAÇÕES DO PENSAMENTO ALGÉBRICO NA ESCOLA BÁSICA}

Iniciamos com Usiskin (1999), que identifica a spectos d o P ensamento A lgébrico destacando que "os propósitos que temos para ensinar Álgebra, as concepções que temos sobre o assunto e os usos das variáveis estão inextricavelmente relacionados." (USISKIN, 1999, p.9)³. Tais propósitos são correlacionados pelo autor a quatro concepções de Álgebra: Álgebra como aritmética generalizada; Álgebra como o estudo de procedimentos para resolver certos tipos de problemas; Álgebra como o estudo de relações entre quantidades; Álgebra como o estudo de estruturas.

Cada uma dessas concepções relaciona-se a usos diferentes da linguagem simbólica e das variáveis e, consequentemente, a aspectos diferentes do Pensamento Algébrico.

Na primeira concepção, Álgebra como aritmética generalizada, os símbolos algébricos são variáveis utilizadas como tradutoras e generalizadoras de padrões, regularidades e resultados. Por exemplo, ao traduzir a sequência descrita como "o dobro de um número adicionado a

\footnotetext{
${ }^{3}$ Tradução nossa de: "[ ...] the purposes we have for teaching algebra, the conceptions we have of the subject, and the uses of variables are inextricably related.” (USISKIN, 1999, p. 9).
} 
sete unidades" para a expressão $(2 x+7)$, a variável x é utilizada como tradutora. Nos livros didáticos brasileiros, um exercício comum sobre generalização de padrões e busca de regularidades é enunciado como se segue:

Dada a sequência abaixo desenhada na Figura 1, encontre as figuras que se encontram nas posições 5, 6 e 9. Depois tente descobrir o padrão que relaciona a posição das figuras às quantidades de bolinhas que as formam. Escreva esse padrão para uma figura qualquer que esteja na posição $n$.

\section{Figura 1 - Sequência de bolinhas}

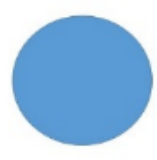

1

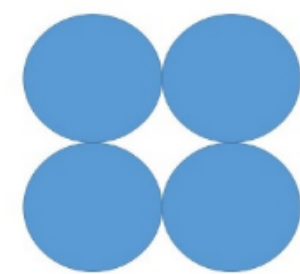

2

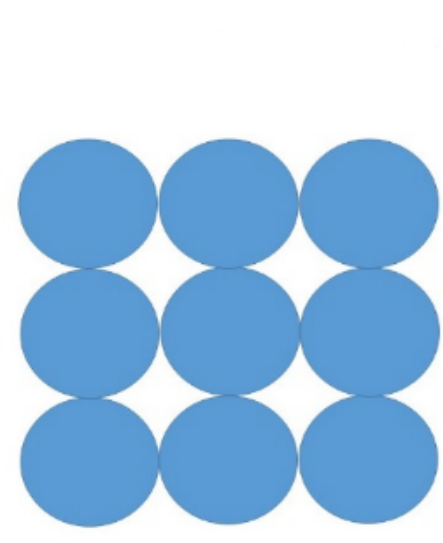

3

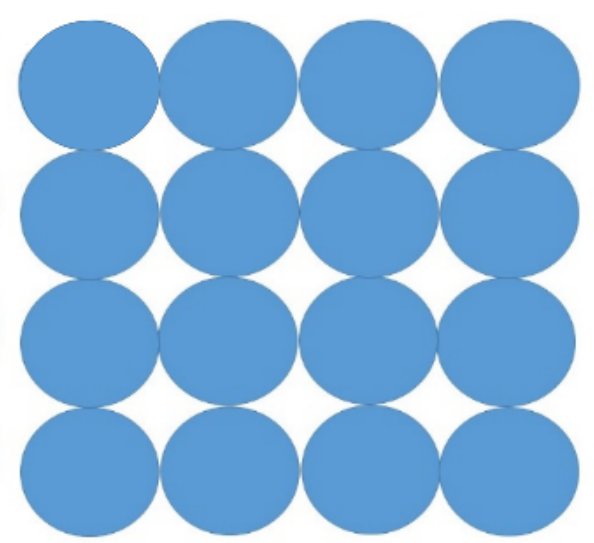

4

Fonte: Arquivo próprio.

Na segunda concepção, descrita como estudo de meios para resolver certos tipos de problemas, os símbolos ou letras são constantes genéricas, usadas como desconhecidas ou constantes, para simplificar expressões e resolver e quações. Por exemplo, quando introduzimos o "quadradinho" para descobrir valores em igualdades entre duas expressões.

No estudo de relações entre quantidades, as variáveis são usadas como parâmetros ou argumentos para relacionar e desenhar gráficos. Por exemplo, ao escrever o preço de certo tipo de fruta em função da quantidade comprada, simbolizamos uma relação entre quantidades.

O uso de variáveis como signos arbitrários, para manipular segundo convenções sintáticas e para justificar resultados, é c aracterístico do conceito de Álgebra como e strutura. Como exemplos na escola básica, temos o estudo de monômios, polinômios, produtos notáveis e fatoração, que generalizam as estruturas operatórias dos números reais. Matrizes e a Álgebra de matrizes, com a definição das operações de adição e multiplicação, são também incluídas aqui.

Destacamos a tradução e generalização de padrões e regularidades, utilização e operação com termos desconhecidos e justificação como características importantes presentes no Pensamento Algébrico.

Há alternativas ao entendimento em Usiskin (1999) sobre as características do Pensamento Algébrico, que o complementam e não necessariamente se opõem a ele, apresentadas por outros pesquisadores. Dentre as referências constantes nas pesquisas sobre Pensamento Algébrico e ensino de Álgebra na escola básica, discutimos elaborações em Kaput (2000), Kaput (2008), Canavarro (2007), Lins e Kaput (2004), Radford (2014), Radford (2009) e Lins (1992). 
Kaput (2000), assim como Oliveira e Laudares (2015), critica abordagens de Álgebra na escola básica que se reduzem a "simplificar as expressões algébricas, resolver equações, aprender as regras para a manipulação de símbolos” (p.2). Para Kaput (2000), é preciso encontrar alternativas para levar os alunos a aprenderem compreendendo, refletindo sobre e articulando conhecimentos e não somente memorizando procedimentos para resolver exercícios e produzir uma resposta. Para ele, o ensino da Álgebra deve:

Começar cedo (em parte, com base no conhecimento informal dos alunos); Integrar a aprendizagem da Álgebra com a aprendizagem de outros assuntos (estendendo e aplicando o conhecimento matemático); Incluir as várias formas diferentes de Pensamento Algébrico (aplicando o conhecimento matemático); Construir sobre os poderes linguísticos e cognitivos naturais dos alunos (encorajando-os, ao mesmo tempo, a refletir sobre o que aprendem e a articular o que sabem) e Encorajar a aprendizagem ativa (e a construção de relacionamentos) que dê uma vantagem na sensação e compreensão. (KAPUT, 2000, p.3) ${ }^{4}$.

Na visão de Kaput (2000), para que tal mudança aconteça no ensino da Álgebra, é preciso propor atividades que envolvam generalizações já no ensino da aritmética - a aritmética generalizada abordada por Usiskin (1999), na modelagem, na geometria, e que passem a ser gradativamente expressas em linguagem formal. Desse modo, o autor argumenta que formas de Pensamento Algébrico, tais como as requeridas em generalizações devem permear todo o currículo de Matemática desde os primeiros anos de escolaridade. O discurso em Canavarro (2007) sobre a "algebrização do currículo" (p.92) retoma ambas perspectivas em Usiskin (1999) e em Kaput (2000).

Complementando os estudos em Usiskin (1999) e indo além da discussão em Kaput (2000), Kaput (2008) identifica elementos do Pensamento Algébrico destacando primeiramente três modos diferentes com que a Álgebra se apresenta em sala de aula na escola. Argumenta que o Pensamento Algébrico envolve dois aspectos centrais - simbolizar generalizações de padrões e restrições, raciocinar e agir sintaticamente ${ }^{5}$ sobre elas quando expressas em simbologia convencional.

Em sua visão, tais aspectos descrevem a Álgebra em três vertentes:

\footnotetext{
${ }^{4}$ Tradução nossa de: “[...] begin early (in part, by building on students' informal knowledge); integrate the learning of algebra with the learning of other subject matter (by extending and applying mathematical knowledge); include the several different forms of algebraic thinking (by applying mathematical knowledge); build on students' naturally occurring linguistic and cognitive powers (encouraging them at the same time to reflect on what they learn and to articulate what they know), and encourage active learning (and the construction of relationships) that puts a premium on sense-making and understanding. (KAPUT, 2000, p.3).

${ }^{5}$ Agir sintaticamente é agir de acordo com as regras da linguagem matemática.
} 
1. Álgebra como o estudo de estruturas e sistemas extraídos de cálculos e relações, incluindo aqueles que surgem em aritmética (Álgebra como aritmética generalizada) e raciocínio quantitativo.

2. Álgebra como o estudo de funções, relações e variação conjunta.

3. Álgebra como a aplicação de um conjunto de linguagens de modelagem dentro e fora da Matemática. (KAPUT, 2008, p.11). ${ }^{6}$

Entendemos que, para esse autor, o Pensamento Algébrico está intrinsecamente relacionado aos processos de generalização, uma vez que explicita tanto o foco em sua representação, quanto a manipulação dos símbolos com os quais generalizações foram expressas. Confirmando esse entendimento, citamos Lins e Kaput (2004), que explicitam uma relação entre aritmética e Álgebra, propondo que o Pensamento Algébrico se revela por meio de "atos de generalização deliberada e expressão da generalidade e também do raciocínio baseado nas formas de generalização sintaticamente estruturados, incluindo ações sintáticas e semânticas.” (LINS; KAPUT, 2004, p.48) ${ }^{7}$.

Kaput (2008) prossegue destacando como elementos do Pensamento Algébrico correspondentes aos três modos ou vertentes com que a Álgebra é apresentada nas escolas, os processos que envolvem a aritmética generalizada (1), o pensamento funcional (2) e a modelagem (3).

Assim como Usiskin (1999) e Kaput (2008), Radford (2009), Radford (2014) também discute caracterizações do Pensamento Algébrico na escola básica. Segundo Radford (2009), o Pensamento Algébrico é a apropriação da abstração algébrica, distanciada do contexto. Essa apropriação se dá por etapas de desenvolvimento sequenciais:

(a) Pensamento Algébrico factual - mais relacionado à aritmética;

(b) Pensamento Algébrico contextual - mais relacionado à expressão de relações através da linguagem natural e à estrutura do contexto;

(c) Pensamento Algébrico padrão - relacionado à síntese da linguagem natural e à estrutura do contexto.

Tais aspectos são retomados em Radford (2014) ao apresentar o resultado de sua pesquisa desenvolvida sobre Pensamento Algébrico na escola básica ao longo de cinco anos, envolvendo crianças do $2^{\circ}$ ao $4^{\circ}$ ano do primeiro ciclo. Segundo ele, o Pensamento Algébrico constitui-se relacionando as três condições a seguir:

\footnotetext{
${ }^{6}$ Tradução nossa de: "1. Algebra as the study of structures and systems abstracted from computations and relations, including those arising in arithmetic (algebra as generalized arithmetic) and in quantitative reasoning. 2. Algebra as the study of functions, relations, and joint variation. 3. Algebra as the application of a cluster of modeling languages both inside and outside of mathematics."(KAPUT, 2008, p.11).

${ }^{7}$ Tradução nossa de: "Second, it involves, usually as a separate endeavour, reasoning based on the forms of syntactically-structured generalisations, including syntactically and semantically guided actions." (LINS; KAPUT, 2004, p.48)
} 
1. indeterminação: [o estudante percebe que] o problema envolve números desconhecidos (incógnitas, variáveis, parâmetros, etc.);

2. denotação: os números indeterminados envolvidos no problema têm de ser nomeados ou simbolizados. [...] Pode-se usar sinais alfanuméricos [...] pode ser simbolizada através da linguagem natural, gestos, sinais não convencionais, ou mesmo uma mistura destes;

3. analiticidade: as quantidades indeterminadas são tratadas como se fossem números conhecidos. Ou seja, embora não sejam conhecidos, parte-se das quantidades indeterminadas e opera-se sobre elas (isto é, acrescenta, subtrai, multiplica, divide) como se elas fossem conhecidas: Isto é o que significa analiticidade. (RADFORD, 2014, p.260 - grifo nosso). ${ }^{8}$

O pesquisador argumenta que o Pensamento Algébrico não necessariamente deve ser estabelecido de modo formal. Propõe que ele pode ser desenvolvido mais cedo, de modo não formal, em atividades envolvendo sequências e padrões para elaborar generalizações. Os resultados de sua pesquisa destacam que os alunos iniciam o processo de desenvolvimento com um pensamento aritmético ${ }^{9}$, depois passam a um Pensamento Algébrico não formal, e só então utilizam a linguagem simbólica, mais formalizada.

Por sua vez, Lins (1992) considera que pensar algebricamente é construir significados e, para essa construção acontecer, há três estágios de pensamento a serem desenvolvidos:

(a) o pensar aritmético - base do pensamento Algébrico e envolve testar e efetuar cálculos de modo a caminhar para uma generalização. Nesse estágio, os número são utilizados como ferramenta. Podemos realizar esse estágio de pensamento com a aritmética generalizada citada em alguns trabalhos aqui pesquisados como Usiskin (1999) e Kaput (2008);

(b) o pensar internalista - é um estágio de pensamento posterior ao pensar aritmético. Inicia-se com um processo de transformação das ferramentas em objeto. Nessa fase, não há necessidade de um contexto para alguma problemática, as ferramentas se tornam objetos matemáticos com os quais já se sabe fazer manipulações, porém, com compreensão de significados, em conformidade com Kaput (2000);

(c) o pensar analítico - é manipular os termos desconhecidos como se fossem conhecidos, como afirma Radford (2014).

\footnotetext{
${ }^{8}$ Tradução nossa de: (1) indeterminacy: the problem involves not-known numbers (unknowns, variables, parameters, etc.); (2) denotation: the indeterminate numbers involved in the problem have to be named or symbolised. Now this symbolisation may be accomplished in various ways. One can use alphanumeric signs-but not necessarily. The denotation of indeterminate quantities can also be symbolised through natural language, gestures, unconventional signs, or even a mixture of these; (3) analyticity: the indeterminate quantities are treated as if they were known numbers. That is, although the are not known, one starts from the indeterminate quantities and operates on them (i.e., adds, subtracts, multiplies, divides them) as if they were known: This is what analycity means. (RADFORD, 2009, p.260)

${ }^{9}$ Segundo o autor, o pensamento aritmético está baseado nas estratégias de tentar e adivinhar, mas não na analiticidade, como o Pensamento Algébrico.
} 
Para Lins (1992), ao desenvolvermos esses três estágios de pensamento, teremos desenvolvido o Pensamento Algébrico.

A próxima seção discute o uso dos elementos trazidos pelos autores referenciando pesquisas sobre o tema na área de Educação Matemática.

\section{CONTRIBUIÇÕES DE PESQUISAS E ORIENTAÇÕES REFERENCIADAS NAS CA- RACTERIZAÇÕES ANTERIORES}

Ferreira (2014) traz resultados de pesquisa sobre a Álgebra na escola e na formação do professor optando por não conceituar Pensamento Algébrico. No entanto, destaca a generalização e os processos de justificação c omo c aracterísticos da Álgebra d a e scola básica, entendendo-os, portanto, como componentes do conteúdo a serem, de algum modo, trabalhados em sala de aula, em consonância com Usiskin (1999) e Kaput (2000). O que consideramos importante é que Ferreira (2014) entende e reafirma que o Pensamento A lgébrico n a escola básica "pode se manifestar e se desenvolver sem a utilização da linguagem algébrica padrão e, portanto, ele pode ocorrer desde os primeiros anos de escolarização." (FERREIRA, 2014, p.45), ou seja, pode ser construído "sobre os poderes linguísticos e cognitivos naturais dos alunos [...]" (KAPUT, 2000, p.3) ${ }^{10}$, por meio de atividades sobre padrões e regularidades, desde os primeiros anos escolares, e prosseguir de modo que essa linguagem natural seja gradativamente formalizada no decorrer da aprendizagem (RADFORD, 2014).

Ao referir-se ao ensino da Álgebra na escola, a autora aponta, ainda, para a formação dos professores e para a necessidade de se conhecerem formas de argumentação e demonstração válidas, que possam ser desenvolvidas no contexto da sala de aula, e que diferem das argumentações e demonstrações que encontramos no contexto do ensino superior. Ferreira (2014) retoma Stylianides e Ball (2008), que consideram que uma demonstração:

(1) Utiliza afirmações, aceitas pela comunidade da sala de aula (conjunto de afirmações aceitáveis), que são verdadeiras e disponíveis sem necessidade de apresentação de justificativas; (2) Emprega formas de raciocínio (modos de argumentação) que são válidas e conhecidas ou dentro do alcance conceitual da comunidade da sala de aula; (3) É passível de ser comunicada por formas de expressão (modos de representação da argumentação) que são próprias e conhecidas ou dentro do alcance conceitual da comunidade da sala de aula. (STYLIANIDES; BALL, 2008, p.309). ${ }^{11}$

${ }^{10}$ Tradução nossa de:“build on students'naturally occurring linguistic and cognitive powers [...]" (KAPUT, 2000, p.3).

${ }^{11}$ Tradução nossa de: (i) it uses statements accepted by the classroom community (set of accepted statements) that are true and available without further justification; (ii) it employs forms of reasoning (modes of argumentation) that are valid and known to, or within the conceptual reach of, the classroom community; and (iii) it is communicated with forms of expression (modes of argument representation) that are appropriate and known to, or within the conceptual reach of, the classroom community. (STYLIANIDES; BALL, 2008, p.309). 
Esses critérios, de fato, que podem ser propostos tanto para a Matemática trabalhada em nível universitário quanto em nível escolar, podem ser úteis para embasar como válidos os pensamentos e raciocínios dos alunos na escola básica ao lidar com questões de generalizações e justificações de maneira não formal, utilizando sua linguagem natural. Argumentação Matemática também é foco de pesquisa em Matos e Ponte (2008), que se referenciam em Kaput (2000) ao entender que:

o Pensamento Algébrico surge quando, através de processos de conjectura e argumentação, se estabelecem generalizações sobre dados e relações Matemáticas, expressas através de linguagens cada vez mais formais. (MATOS; PONTE, 2008, p.197).

Por "cada vez mais formais", entendemos que as linguagens não necessariamente têm origem formal, podem ser não formais, porém, vão sendo formalizadas à medida que o estudante amadurece matematicamente ${ }^{12}$. Matos e Ponte (2008) investigaram as relações funcionais - Álgebra como o estudo de relações entre quantidades (KAPUT, 2008; USISKIN, 1999) - e o desenvolvimento do Pensamento Algébrico em alunos do $8^{\circ}$ ano, dando especial atenção ao modo como eles interpretam e utilizam a linguagem algébrica. Concluíram que a ênfase em atividades sobre relações funcionais promoveu o desenvolvimento de significado para a linguagem algébrica, e a construção de uma visão mais ampla sobre o uso de símbolos.

Canavarro (2007) também desenvolve pesquisa empírica ilustrando suas ideias teóricas com episódios de sala de aula, envolvendo estudantes de sete e oito anos do $1^{\circ}$ e $2^{\circ}$ ciclos em Portugal. Discute "a pertinência da inclusão do Pensamento Algébrico no currículo de Matemática dos primeiros anos" (p. 81) por meio de busca por padrões e regularidades iniciados com aritmética. Prossegue com a tentativa de generalizar os padrões descobertos (KAPUT, 2000), utilizando a linguagem natural dos alunos e formalizando-a no decorrer do tempo (MATOS; PONTE, 2008; RADFORD, 2014).

Da análise dos episódios descritos em seu trabalho, a autora destaca aspectos sofisticados do raciocínio matemático que crianças de sete e oito anos manifestaram:

Identificaram a e strutura $\mathrm{M}$ atemática d a s ituação e $\mathrm{m}$ a nálise; estabeleceram relações numéricas entre as duas variáveis em causa; generalizaram uma regra para a determinação de qualquer termo da sequência, em linguagem natural, justificando-a; expressaram a generalização de duas formas distintas, por recorrência e através do termo geral. (CANAVARRO, 2007, p.86).

Para a autora, a generalização é o foco do Pensamento Algébrico e a Álgebra deve ser estudada de modo a enfatizar significados e compreensão, concordando com Kaput (2000). A questão não é somente focar os símbolos, mas sim enxergar a partir deles.

No entanto, "as abordagens, tradicionalmente difundidas em torno da Álgebra têm colocado em foco principalmente a memorização e mecanização de fórmulas, como metodologia

\footnotetext{
${ }^{12}$ Com a expressão "amadurece matematicamente", referimo-nos a estágios de aquisição gradativa de conhecimento e de desenvolvimento de habilidades de manipulação simbólica, de incorporação de modos de representação utilizando linguagem matemática e representações simbólicas.
} 
para assimilação dos conceitos algébricos" (OLIVEIRA; LAUDARES, 2015, p.2), sem que o aluno entenda o que está sendo feito. Concordamos com Canavarro (2007) que tais aulas mecânicas, "centradas no modelo de explicação por parte do professor seguida de aplicação e treino por parte dos alunos, não são um contexto favorável ao desenvolvimento do Pensamento Algébrico." (CANAVARRO, 2007, p.114).

Contrapondo-se a esse ensino mecânico, Canavarro sugere uma "algebrização do currículo" (CANAVARRO, 2007, p.92), ou seja, um currículo totalmente permeado por abordagens algébricas desde os primeiros anos de escolaridade, relacionando esse pensamento com outros conteúdos da Matemática, e visando aprimorar as habilidades e competências cognitivas e linguísticas dos alunos. Em sua visão, a introdução da Álgebra desde os primeiros anos:

inspira uma abordagem [...] na qual os alunos desenvolvam as suas capacidades matemáticas [...] (constroem) conhecimento relevante, com compreensão, [...] (e assim conseguem) melhorar a preparação para as aprendizagens posteriores. (CANAVARRO, 2007, p.113, grifo nosso).

Por fim, não podemos deixar de mencionar Kieran (2004), que realizou sua pesquisa tendo como proposta uma definição para Pensamento Algébrico na escola básica, com foco na aprendizagem de Álgebra envolvendo estudantes de 12 a 16 anos. Em Québec no Canadá, essa faixa etária refere-se ao Secondary Education, equivalente ao nosso Ensino Médio, porém, com suas particularidades ${ }^{13}$. A autora discutiu as diferenças entre os pensamentos aritmético e algébrico e elaborou os principais componentes da atividade algébrica.

Segundo Kieran (2004, p.140), "[...] os alunos que operam em um quadro de referência aritmético tendem a não ver os aspectos relacionais das operações; o foco deles é o cálculo."14. Assim, o pensamento aritmético traz implícita a ação. Por exemplo, ao trabalhar com o "valor do quadradinho" 15 no Ensino Fundamental I, o aluno consegue com a ação de "desfazer", ou por meio de tentativas, descobrir o numeral que deve ser colocado dentro do quadradinho. Porém, ao iniciar o estudo de equações, quando incógnitas são introduzidas, o aluno não consegue perceber o significado trazido pela equação, pois ele está acostumado com a ação e não com o pensar significados. A pesquisadora acredita que significados pode aflorar com o desenvolvimento do Pensamento Algébrico desde os anos iniciais, como defendido até aqui.

Dessa forma, é necessário repensar abordagens para problemas, a fim de promover:

\footnotetext{
${ }_{13}$ Para mais informações sobre o quadro escolar canadense, veja: http://www.planetinterchange.com/blog-epromocoes/blog/como-funciona-a-escola-no-quebec.

${ }^{14}$ Tradução nossa de "[...] students operating in an arithmetic frame of reference tend not to see the relational aspects of operations; their focus is on calculating." (KIERAN, 2004, p.140)

${ }^{15}$ Encontrar o valor do quadradinho é atividade do tipo: $2+[]=10$.
} 
[...] um ajuste considerável no desenvolvimento de uma forma de Pensamento Algébrico, que inclui, mas não se restringe a:

1. Um foco nas relações e não apenas no cálculo de uma resposta numérica;

2. Um foco nas operações, bem como em seus inversos, e na ideia relacionada de fazer/desfazer;

3. Um foco em representar e resolver um problema em vez de simplesmente resolvê-lo;

4. Um foco em números e letras, em vez de em números sozinhos. Isso inclui:

I trabalhar com letras que às vezes podem ser desconhecidas, variáveis ou parâmetros;

II aceitar expressões literais não fechadas como respostas;

III comparar expressões para equivalência com base em propriedades e não na avaliação numérica;

5. Uma reorientação do significado do sinal de igualdade. (KIERAN, 2004, p.140-141). ${ }^{16}$

Podemos considerar que a introdução desses cinco focos, apontados por Kieran (2004), desde as séries iniciais, colaborariam com o desenvolvimento de formas de pensar para propor modelagens, resoluções, generalizações e argumentações, sendo assim um facilitador ao entendimento com compreensão de uma Álgebra mais avançada e formal nas séries posteriores. Ou seja, a Álgebra estudada nas séries mais avançadas se tornaria mais acessível para a maioria dos estudantes.

Por fim, Kieran propõe o seguinte entendimento para Pensamento Algébrico:

O Pensamento Algébrico nas séries iniciais envolve o desenvolvimento de formas de pensar nas atividades para as quais a Álgebra simbólica pode ser usada como uma ferramenta, mas que não são exclusivos da Álgebra e que podem ser envolvidos sem usar qualquer Álgebra simbólica de letras, tais como, analisando relações entre quantidades, observando estruturas, estudando mudanças, generalizando, resolvendo problemas, modelando, justificando, provando e prevendo. (KIERAN, 2004, p.149) ${ }^{17}$

\footnotetext{
${ }^{16}$ Tradução nossa de "[...] considerable adjustment is required in developing an algebraic way of thinking, which includes, but is not restricted to: 1. A focus on relations and not merely on the calculation of a numerical answer; 2. A focus on operations as well as their inverses, and on the related idea of doing / undoing; 3 . A focus on both representing and solving a problem rather than on merely solving it; 4 . A focus on both numbers and letters, rather than on numbers alone. This includes: (i) working with letters that may at times be unknowns, variables, or parameters; (ii) accepting unclosed literal expressions as responses; (iii) comparing expressions for equivalence based on properties rather than on numerical evaluation; 5. A refocusing of the meaning of the equal sign."(KIERAN, 2004, p.140-141).
} 
Nessa caracterização, podemos observar que a autora fala sobre a utilização da linguagem natural como em (CANAVARRO, 2007; FERREIRA, 2014; RADFORD, 2009; KAPUT, 1999), “[...] sem usar qualquer álgebra simbólica de letras [...]”, e sobre pensamento funcional, como em (MATOS; PONTE, 2008; USISKIN, 1999), “[...] analisando relações entre quantidades [...]" e, estudo de variações e estruturas, generalizações, modelagens e justificações, como em (KAPUT, 2008; CANAVARRO, 2007; FERREIRA, 2014). Em outras palavras, há acordos e complementaridade entre os elementos destacados pelos diversos autores ao caracterizar o Pensamento Algébrico na escola básica.

A partir do estudo realizado sobre elementos, que alguns pesquisadores em Educação Matemática e educadores identificam c omo i mportantes a o d esenvolvimento do Pensamento Algébrico do estudante na escola básica, queremos destacar, em especial habilidades, sugeridas a partir de Radford (2014), que são compartilhadas por muitos dentre os outros pesquisadores que estudamos: a habilidade de entender um problema, perceber a existência de valores desconhecidos que, por vezes, podem ser encontrados usando a noção de incógnita a ser determinada e de resolução de equações; de generalizar padrões aritméticos fazendo uso das propriedades das operações aritméticas e da noção de constantes genéricas; de expressar relações e funções em processos de modelar um fenômeno ou problema, trazendo a noção de variação de grandezas e de variáveis; de fixar relações, argumentar, utilizar a linguagem simbólica abstratamente, manipular analiticamente os símbolos, como fatores em cálculos algébricos, obtendo expressões equivalentes. Como uma síntese possível, tais habilidades podem ser relacionadas com as dimensões ou concepções da Álgebra propostas em Usiskin (1999): a Álgebra como aritmética generalizada; a Álgebra como o estudo de procedimentos para resolver certos tipos de problemas; a Álgebra como o estudo de relações entre quantidades; a Álgebra como o estudo de estruturas.

Vale mencionar a análise posterior do texto dos Parâmetros Curriculares Nacionais (PCN) com o objetivo de relacionar a literatura de pesquisa e as diretrizes curriculares nacionais. Reportamos na Tabela 1, a tabela síntese referente à Matemática do Ensino Fundamental 2, que consideramos importante trazer aqui.

\footnotetext{
${ }^{17}$ Algebraic thinking in the early grades involves the development of ways of thinking within activities for which letter-symbolic algebra can be used as a tool but which are not exclusive to algebra and which could be engaged in without using any letter-symbolic algebra at all, such as, analyzing relationships between quantities, noticing structure, studying change, generalizing, problem solving, modeling, justifying, proving, and predicting.
} 
Tabela 1 - Dimensões da Álgebra, uso de letras e conceitos

\begin{tabular}{|c|c|c|c|c|}
\hline \multicolumn{5}{|c|}{ Álgebra no Ensino Fundamental } \\
\hline $\begin{array}{c}\text { Dimensões da } \\
\text { Álgebra }\end{array}$ & $\begin{array}{c}\text { Aritmética } \\
\text { Generalizada }\end{array}$ & Funcional & Equações & Estrutural \\
\hline Uso das Letras & $\begin{array}{l}\text { Letras como } \\
\text { generalizações do } \\
\text { modelo aritmético }\end{array}$ & $\begin{array}{l}\text { Letras como } \\
\text { variáveis para } \\
\text { expressar } \\
\text { relações e } \\
\text { funções }\end{array}$ & $\begin{array}{c}\text { Letras como } \\
\text { incógnitas }\end{array}$ & $\begin{array}{c}\text { Letras como } \\
\text { símbolos } \\
\text { abstratos }\end{array}$ \\
\hline $\begin{array}{c}\text { Conteúdos } \\
\text { (conceitos e } \\
\text { procedimentos) }\end{array}$ & $\begin{array}{l}\text { Propriedades das } \\
\text { operações } \\
\text { Generalizações de } \\
\text { padrões aritméticos }\end{array}$ & $\begin{array}{l}\text { Variação de } \\
\text { grandezas }\end{array}$ & $\begin{array}{l}\text { Resolução } \\
\text { de equações }\end{array}$ & $\begin{array}{c}\text { Cálculo } \\
\text { algébrico } \\
\text { Obtenção de } \\
\text { expressões } \\
\text { equivalentes }\end{array}$ \\
\hline
\end{tabular}

Fonte: Adaptado do quadro da página 116, Parâmetros Curriculares Nacionais (BRASIL, 2000).

Essa tabela organiza vários aspectos identificados e discutidos em nosso e studo. A referência a diferentes dimensões da Álgebra, aos diferentes significados das "letras" utilizadas em Álgebra, e aos conceitos trabalhados em cada uma dessas dimensões, está em diálogo com os autores estudados e oferece um modo de organizar os aspectos elencados a partir da revisão de literatura. Essa síntese torna-se importante para orientar nossa própria caracterização de pensamento algébrico na escola básica, bem como a elaboração de atividades para a sala de aula, trazendo as nuances e distinções de que nem sempre somos conscientes, uma vez já tendo incorporado adequadamente o trabalho com a Álgebra.

\section{CONSIDERAÇÕES FINAIS: NOSSA CARACTERIZAÇÃO DE PENSAMENTO ALGÉBRICO}

De acordo com os autores estudados, para desenvolver o Pensamento Algébrico, é preciso aprender compreendendo, pensar com compreensão, produzir significados, e nxergar por meio dos símbolos. Em sua maioria, esses autores concordam que tal pensamento é alcançado de modo progressivo, em etapas que se sucedem até que o Pensamento Algébrico seja desenvolvido. Em sua forma algébrico-simbólica, a força está em seu distanciamento do contexto e no significar objetos de u ma maneira abstrata (RADFORD, 2014). Esse processo de desenvolvimento do Pensamento Algébrico não é algo que acontece naturalmente, pois ele "é um tipo de reflexão e ação cultural muito sofisticado, um modo de pensamento que foi refinado sucessivamente ao longo de séculos antes de alcançar sua forma atual." (RADFORD, 2011 apud ALMEIDA; SANTOS, 2017).

A partir das pesquisas e reflexões desses autores, entendemos que o Pensamento Algébrico é uma forma de pensamento matemático que incorpora em si diversas habilidades, tais 
como: a habilidade de entender um problema, perceber a existência de valores desconhecidos e padrões, de generalizar, de modelar, de fixar relações, de argumentar matematicamente, utilizar a linguagem simbólica e manipular analiticamente. ${ }^{18}$ Assim, desenvolver o Pensamento Algébrico dos estudantes pode ser entendido como atuar de modo a fazer emergir tais características ao abordar problemas matemáticos. O objetivo é desenvolver significados para uma linguagem que é utilizada e não somente a utilizar sem saber o que significa.

Pesquisas produzidas sobre pensamento algébrico nas últimas décadas, em sua maioria, defendem um ensino de álgebra o mais cedo possível. Não o de uma álgebra formal, simbólica, ou de aplicação de regras e fórmulas; mas sim, de um ensino de Álgebra na escola básica que permita o desenvolvimento do raciocínio, do Pensamento Algébrico. Esse ensino tem sido proposto através de reconhecimento e extensão de padrões, partindo de uma base aritmética e indo além. Sem demandar formalizações excessivas, pode-se utilizar a linguagem natural, gestos, entre outros.

Os autores citados investigaram ainda o desenvolvimento do Pensamento Algébrico que tem origem em argumentações, envolvendo saber pensar reflexivamente. Mostrando, dessa forma, a importância de desenvolver o Pensamento Algébrico para o aluno que estuda Matemática.

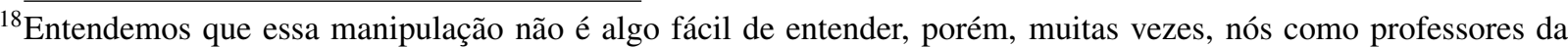
escola básica lecionamos como se isso fosse algo trivial. A essa manipulação chamamos analiticidade e foi introduzida na matemática por François Viète (ROQUE, 2012).
} 


\section{REFERÊNCIAS}

ALMEIDA, J. R.; SANTOS, M. C. Pensamento Algébrico: Em busca de uma definição. Revista Paranaense de Educação Matemática, v. 6, n. 3, 2017.

BRASIL, Secretaria de Educação Média e Tecnológica. Parâmetros Curriculares Nacionais: Ciências da Natureza, Matemática e suas Tecnologias. Brasília: MEC / SEMTEC, 2000.

BRUNER, J. S. Toward a theory of instruction. Cambridge: Belkapp Press, 1996.

CANAVARRO, A. P. O Pensamento Algébrico na Aprendizagem da Matemática nos Primeiros Anos. Quadrante, v. 16, n. 2, 2007.

FERREIRA, M. C. C. Conhecimento Matemático Específico para o Ensino na Educação Básica: A Álgebra na Escola e na Formação do Professor. 2014. Tese (Doutorado em Educação) - Universidade Federal de Minas Gerais, 2015.

HIEBERT, J. (ed). Conceptual and procedural knowledge in mathematics: An introductory analysis. In: HIEBERT, J.; LEFEVRE, P. (Ed.). Conceptual and Procedural Knowledge: The case of Mathematics. Hillsdale: NJ-London: Lawrence Erlbaum Associates, 1986, p. $1-23$.

HOUAISS, A.; VILlAR, M. de S. Dicionário Houaiss da Língua Portuguesa. Rio de Janeiro: Objetiva, 2001.

KAPUT, J. J. Teaching and learning a new algebra in Mathematics classrooms that promote understanding. [S.1.]: Routledge, 1999.

KAPUT, J. J. Teaching and learning a new algebra with understanding. NSF Applications of Advanced Technology Program, University of Massachusetts-Dartmouth, 2000.

KAPUT, J. J. What is algebra? what is algebraic reasoning? In: KAPUT, J. J.; CARRAHER, D. W.; BLANTON, M. L. (Ed.). Algebra in the early grades. New York: Lawrence Erlbaum Associates, 2008.

KIERAN, C. Algebraic thinking in the early grades: What is it? The Mathematics Educator, v. 8, n. 1, p. 139-151, 2004.

LINS, R. A framework for understanding what algebraic thinking is. 1992. Tese (PhD) - University of Nothingam, Nothingam, United Kingdom, 1992. Disponível em: <http: // eprints.nottingham.ac.uk/13227/1/316414.pdf>.

LINS, R.; KAPUT, J. J. The early development of algebraic reasoning: The current state of the field. In: STACEY, K., CHICK, H., KENDAL, M. (Ed.). The Future of the Teaching and Learning of Algebra The 12thICMI Study. Springer, Dordrecht: New ICMI Study Series, 2004, v. 8.

MATOS, A.; PONTE, J. P. da. O estudo de relações funcionais e o desenvolvimento do conceito de variável em alunos do $8^{\circ}$ ano. Revista Latinoamericana de Investigación en Matemática Educativa, v. 11, n. 2, p. 195-231, 2008. 
OLIVEIRA, S. C. de; LAUDARES, J. B. Pensamento algébrico: Uma relação entre álgebra, aritmética e geometria. In: ENCONTRO MINEIRO DE EDUCAÇÃO MATEMATICA (EMEM), 7., 2015, Juiz de Fora: Anais... Juiz de Fora: Universidade Fede-ral de Juiz de Fora, 2015.

RADFORD, L. Signs, gestures, meanings: Algebraic thinking from a cultural semiotic perspective. In: CONGRESS OF THE EUROPEAN SOCIETY FOR RESEARCH IN MATHEMATICS EDUCATION, 6., 2009, Lyon (France). Anais... Lyon: IFE, 2009. Disponível em: <http://ife.ens-lyon. fr/publications/edition-electronique/cerme6/plenary1radford.pdf $>$.

RADFORD, L. The progressive development of early embodied algebraic thinking. Mathematics Education Research Journal, v. 26, n. 2, p. 257-277, 2014.

ROQUE, T. História da matemática, Uma Visão Crítica, Desfazendo Mitos e Lendas. Rio de Janeiro: Zahar, 2012.

STYLIANIDES, A.; BALL, D. Understanding and describing mathematical knowledge for teaching: knowledge about proof for engaging students in the activity of proving. Journal of MathematicsTeacher Education, v. 11, n. 4, p. 307-332, 2008.

USISKIN, Z. Conceptions of school algebra and uses of variables. The ideas of algebra, K-12 Readings from NCTM's School-Based Journals and Other Publications, edited by Barbara Moses, Reston, p. 7-13, 1999. 